\title{
Control of a Robot Painting System Using the Multi-resolution Architectural Principle - A Summary -
}

\author{
Cristina Santos, Nuno Monteiro, Jaime Fonseca, Paulo Garrido, Carlos Couto \\ Department of Industrial Electronics \\ School of Engineering \\ University of Minho \\ Braga \\ Portugal
}

\begin{abstract}
The use of a multi-resolution control model for a robot painting system is reported in this paper. It gives a flexible and general framework for the control system design which eases software development and integration, as well as experimentation with different sensory behaviours.
\end{abstract}

\section{INTRODUCTION}

The main goal of the project described in this paper is to develop a robot system with the capability of painting different parts with on-line definition of the painting trajectories. A robot mounted video camera, ultrasonic and infrared sensors are used to acquire data for internally representing the geometry of the part's surface to be painted, exploiting the mobility of the robot. The generation of the painting trajectories will be subsequently based on the internal representation.

The diverse nature of sensory input, the recognising and planning tasks involved, the link between actuation and sensing, make the design of the control structure for this project a non-trivial problem and call for a relatively sophisticated conceptual base. Multi-resolution, i.e., the decomposition of the envisaged system according to timescales of information processing or granularity of representation, has been gaining increased attention as a principle for the understanding and design of complex systems [1]. In the fields of control and robotics, J. Albus and co-workers [2] [3] [4] applied the multi-resolution principle in a reference model architecture for intelligent control systems. (In the sequel, it will be referred as the RCS model for short.) It has been adopted in this project as a general conceptual framework for global design of the system and software integration.

In the next section, we give brief overviews of the RCS model and how the components of the robot painting system are mapped on it. Sections III and IV describe the software procedures which implement image acquisition and depth map filling of the part to be painted. Section V concludes and lists future work.

\section{OVERVIEW OF THE PROJECT}

The RCS model goes further than multi-resolution in recognising that an intelligent system must be organised in levels according to the time-scales implicit in perception and action or the detail of representation of environment and self. The RCS model also decomposes each level in four functions or modules (see Fig. 1):
- World Modelling (WM), a multi-faceted activity which builds, maintains and allows the use of world representations;

- Sensory Processing (SP), the transformation of data from sensors in elements or structures of WM;

- Behaviour Generation (BG), the planning and control of action designed to achieve behavioural goals;

- Value Judgement (VJ), the evaluation of perceived and planned situations to enable BG to select advantageous goals and select priorities.

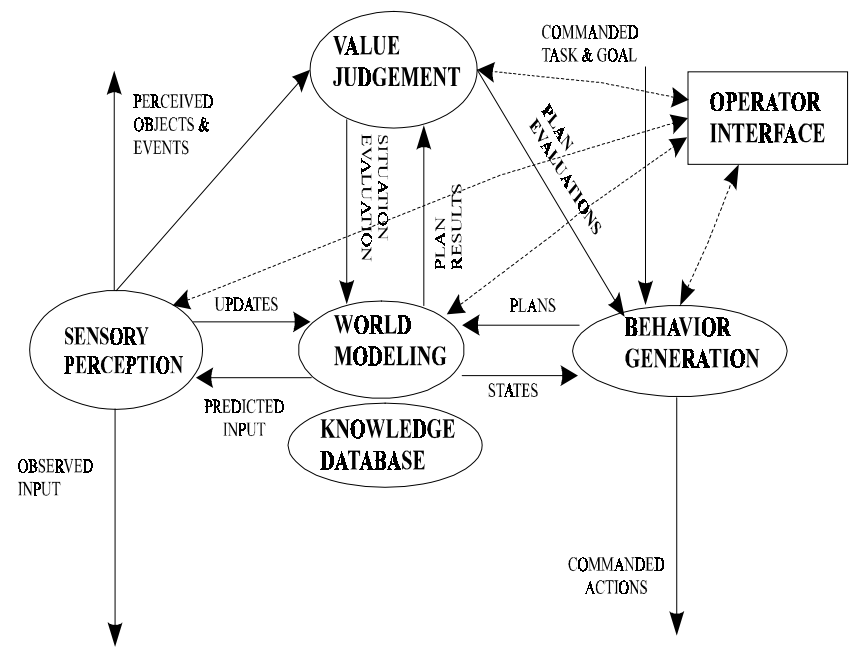

Fig. 1 - Decomposition in modules of each level of a RCS model.

RCS establishes the interactions among these modules in such a way that, globally speaking, each level functions as a feedback arch anchored in the preceding one(s) and allowing the anchoring of the succeeding one. So, in the overall, the system is an hierarchical structure of arches which feedback loop the environment and itself at different levels of speed of answer, width of time window, depth of memory and complexity of relations involved. Let us note that RCS allows for different hierarchical structures to exist in parallel and converge at some pre-specified level and that at each level communication between modules may be bi-directional.

At present, a Puma 560 is being used as the manipulator of the robot painting system. The video camera and one ultrasonic sensor are mounted over the shoulder of the Puma (see Fig. 2 for a general and a detailed view). This ultrasonic sensor is used to locate and align the video camera with the part. 

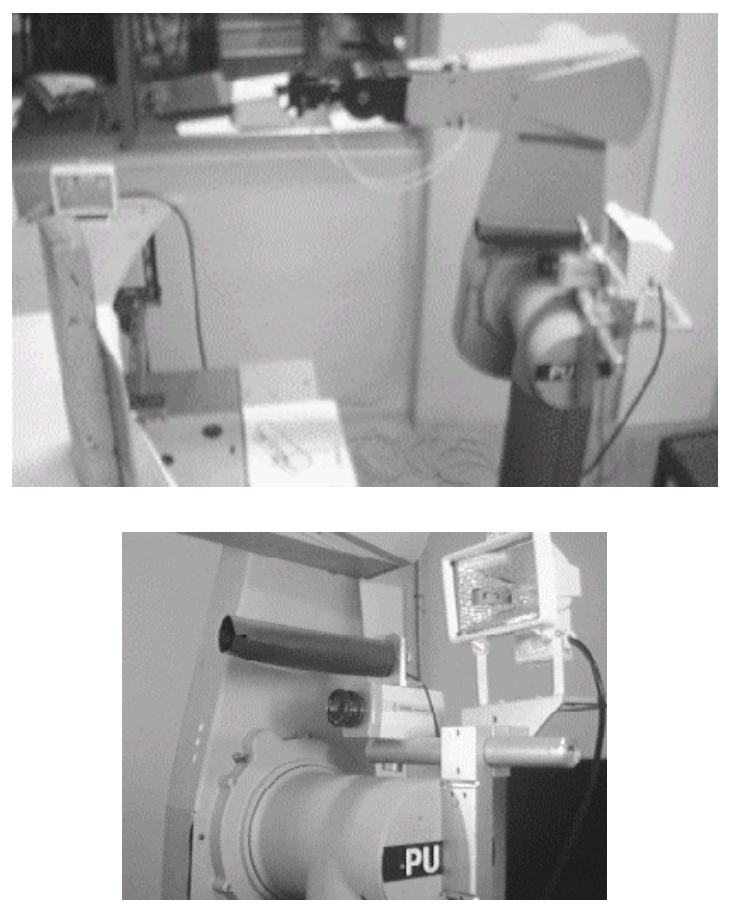

Fig. 2 - Location of the video camera in the robot painting system.

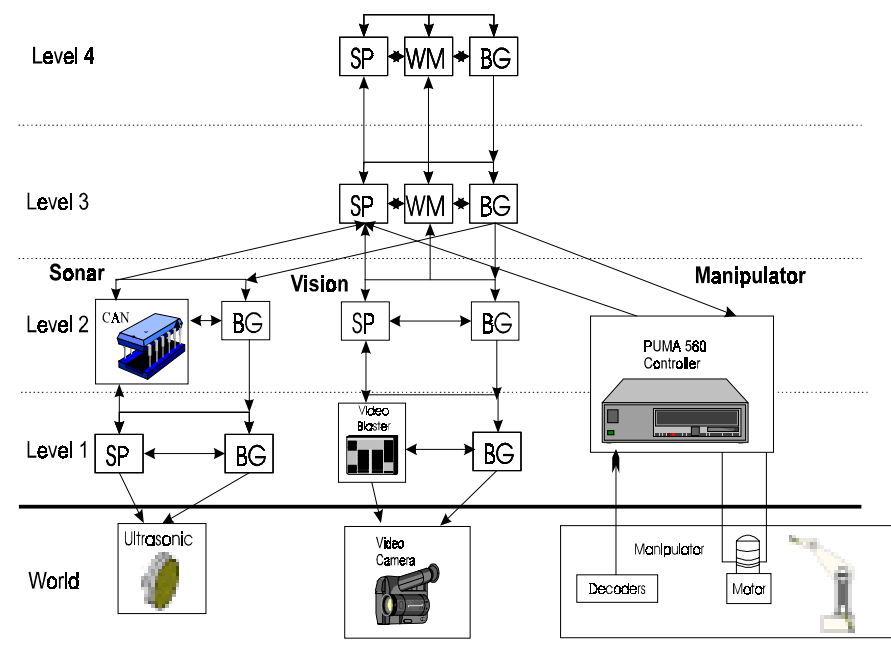

Fig. 3 - The RCS reference model architecture, as applied to the robot painting system.

This set-up allows for confining the work area of the manipulator, acquiring the 2D information (image) of the part's surface in front of the manipulator and approximating its position in the robot co-ordinate system. The 3D (or depth) information will be measured by 4 ultrasonic sensors (range: $35 \mathrm{~cm}$ to $3 \mathrm{~m}$ ) and 4 infrared sensors (range: $10 \mathrm{~cm}$ to $80 \mathrm{~cm}$ ), mounted on a fixture at the wrist of the manipulator. The manipulator will be used as a mobile scanner, to position the depth sensors along a grid of measuring points over the part. This will make for having a map of the projection of the part's surface to be painted, in the manipulator co-ordinate system. Based on this map, the needed painting trajectories will be defined on-line.

The computing hardware includes a CAN (Controller Area Network) board and a PC with a Pentium processor. The data of the ultrasonic and infrared sensors are acquired at the CAN board. The data are pre-processed and sent to the PC via the CAN net at a $1 \mathrm{Mb}$ baudrate. The CCD video camera connects to a low-cost video blaster acquisition board mounted inside the PC. The communication with the Puma is now realised by a serial link to a standard trajectory controller. It is planned to substitute this controller by Trident boards, for enhanced performance.

In Fig. 3 the reader can appreciate the mapping of these elements in a RCS model with four levels and three different hierarchies: vision, sonar and manipulator.

Level 4 acts as a sequencer for the location and map building of the part. As the project advances, more sophisticated functions will be assigned to this level, as adapting the $2 \mathrm{D}$ resolution of the depth scanning or clustering similar shapes of parts for improved speed of the map building process.

At level 3, the SP module allows for creating a map of the part's surface to be painted which constitutes the global WM of this level. At present, the BG module is responsible for, in interaction with SP and WM, aligning the camera with the part, and organise and control the depth scanning sequence which incrementally fills the part's map. It will also be responsible for generating the painting trajectories.

The Puma 560 Controller is distributed over level 2 and 1 and it decomposes trajectory controller commands into the voltages needed to drive the robot's motors. The SP module of the vision hierarchy at level 2, allows to create the dominant points of the surface, while the corresponding in the sonar hierarchy supplies the distance measures.

The image acquisition and the first processing of the sonar measures are done at level 1.

\section{ACQUIRING THE PART'S SURFACE IMAGE}

The vision system is composed by a National Electronics CCD video camera, a low-cost Creative's Video Blaster acquisition board and a set of software procedures developed with Borland $\mathrm{C}++$ under Windows environment.

The assigned goal of this system is to extract the information needed to create a $2 \mathrm{D}$ representation of the part's surface that the system should paint (this is the surface that faces the camera). This 2D representation takes the form of a contour defined by the dominant points. Furthermore, these dominant points are assigned to a set of possible positions in the robot axis system. This will be the basis for the construction of the depth surface map.

Figure 4 illustrates the information extraction process in the vision system.

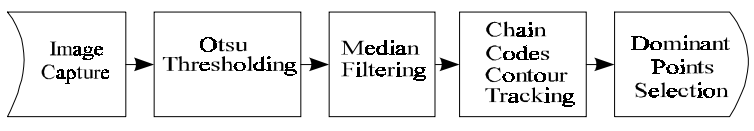

Fig. 4 - Image information extraction process.

After the image has been captured, segmentation is applied. This is achieved by Otsu global thresholding [5], selected on the basis of a comparative study covering Otsu, Maximum Entropy, Uniform Error and Minimum Error threshold selection methods described in [6]. 
After the image has been segmented into object and background regions and the main acquisition noise removed by means of a smoothing median filtering [7], a boundary tracking procedure is applied. This has the purpose of basing the extraction of a set of dominant points meant to represent the 2D shape of the object's image captured.

For the purpose of extracting the dominant points, a combination of two algorithms was used. The first marks pixels as candidates for dominant points and it is an improved version of the classical splitting method presented by Duda and Hart. The second provides the selection and is based on slope. This arrangement was devised to provide a dominant points extraction process suitable for most different sorts of object shapes. An example of the performance of the dominant points extraction process is shown in figure 5.

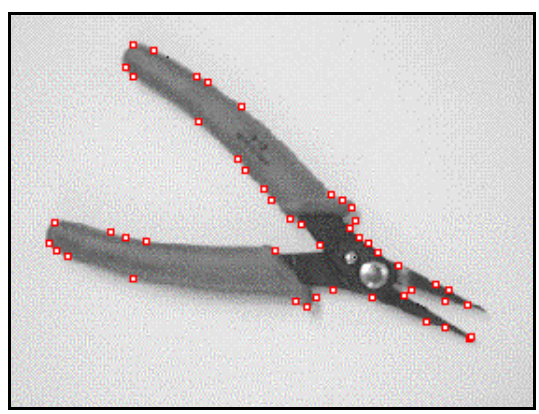

Fig. 5 - Results from the dominant points extraction process superimposed on an original image.

The final task is to compute a first approximation to the dominant points co-ordinates in the robot's system, from their video camera co-ordinates. For this purpose, the distance from the camera to the object, given by the ultrasonic sensor mounted on top of the video camera is used.

\section{DEPTH MAP BUILDING}

A complete representation of the part's surface in robot co-ordinates demands that depth measurements be made in order to obtain 3D information. As said, this is accomplished by making the manipulator scan the 2D shape with its ultrasonic or infrared distance sensors. The aim of having these two types of sensors is to cope with the limitations of each one. At the present stage of the system's development, only ultrasonic sensors are in consideration, and so only them will be referred from now on. The overall result of this task is the building of a surface map which shall support the generation of painting trajectories [8].

Two approaches were experimented for the building of the surface map. In the first - direct depth-map building - a straightforward scanning of the part at a constant angle of the sensors with direct registering of measured values has been used. Problems with sonar distance measures suggested a second approach. In this one - averaged measure weighted by confidence levels - the values assigned to each scanning point are a weighted average of the four sonar measures. This approach given better results, and was easily implemented by transforming the first.

\section{A. Direct depth-map building}

Departing from the output of the SP module of level 2 from the vision hierarchy - a sequence of dominating points defining the part's shape contour -, a general polygon-scan conversion algorithm, which handles convex and concave polygons [9], is used to build a $2 \mathrm{D}$ representation in the form of a linked list of segments of horizontal scan lines belonging to the shape. This algorithm operates by computing spans that lie between left and right edges of the polygon. The span extrema are calculated by an incremental algorithm that computes a scan-line/edge intersection from the intersection with the previous scan line [10].

The disposition of the four sensors, relative to the robot grip's axis, is a square as presented in the Fig.6.

The $y$ co-ordinates that the image acquisition obtained for the part's dominant points, are subjected to perspective errors. Those values were based on the depth measurement given by the sonar mounted on the top of the video camera, which is not the depth of all points but only approximately of the object's centroid. The only co-ordinates that system can consider without error are the $z$ co-ordinates, because it is known that the object is positioned over the table, which has a fixed $z$ co-ordinate. In fact, the true values of the $x, y$ co-ordinates of the boundary points will be defined later by sensor acquisition. So, when acquiring points in the boundary region an uncertainty margin must be considered. For this, the found boundaries are augmented by a certain default value, as illustrated in Fig. 7.

Once the extreme possible values for the boundaries of the object are estimated, the robot is moved to the lowest $z$ co-ordinate and the leftmost $y$ co-ordinate of the shape, at a distance in the range of the ultrasonic sensors and depth scanning begins.

For each scan line, a set of evenly spaced points is defined, all over the surface of the object. The minimal increment between these points is set to the edge's length of the sensors' square arrangement. The robot will step along those points sweeping the $y$ co-ordinates along a fixed $z$ co-ordinate, and then incrementing to the next $z$ coordinate by the same value. The process repeats till all the shape is swept, as illustrated in Fig. 7. The robot's position increment between steps, is twice the distance between

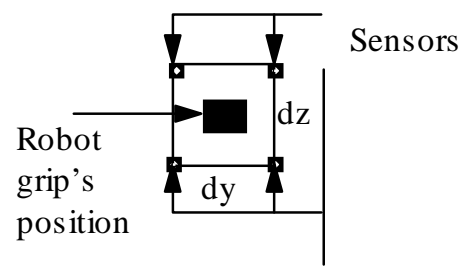

Fig. 6 - Location of the four sensors

sensors. At each step four depth measures are taken and assigned to the points of the part which supposedly are in the axis of each sensor. 


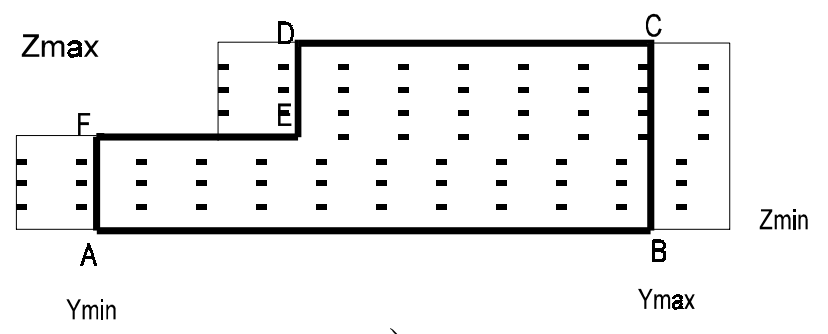

a)

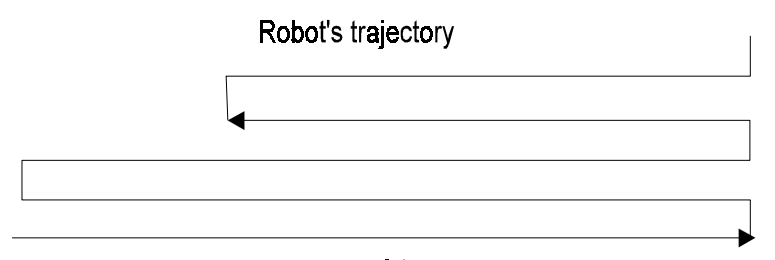

b)

Fig. 7 - Surface acquisition. a) Increment definition along the object's surface and error border - each marked point corresponds to a grip's centre position. b) Robot's trajectory

The experimental results of this process having been applied to a rectangular plane part are displayed in Fig. 8, with the use of Matlab.

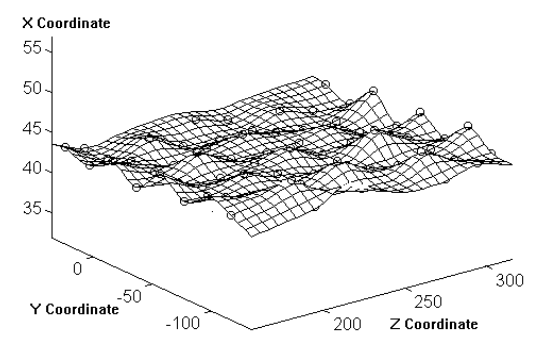

Fig. 8 - Experimental results for the map of a plane rectangular part with direct registering of the four sonar measures

Two problems became evident:

- at the part's border zones, sonar measures become completely unreliable, so the detection of edges positions is inaccurate;

- at the interior of the part significant dispersion among sensors' measures appears.

Obviously, these results call for a more sophisticated sensory behaviour. With a partial change of the modules at the level 3 of the reference architecture, a second approach based on averaging the measures was generated.

\section{B. Averaged measure weighted by confidence levels}

Assuming that the surface does not present significant changes at the scale of the square defined by the four sensors, it seems reasonable to accept that the average of the measure from sensors can be assigned to that point of the part's surface which lies on the grip axis, i.e., in the axis passing through the centre of the square's arrangement of sensors. In doing this, to maintain the resolution of the depth map, speed of acquisition must be traded-off against sensor fusion in order to get greater accuracy.

Besides spatially averaging the sonar measures, weights for the measure of each sensor were introduced. These weights are to be interpreted as degrees of confidence, based on a heuristic set of rules [11].
Let $i d \_s$ be the identifier of a sensor, Measure the measure it gives at a given grip position, $D c$ its actualised degree of confidence. Then $M$, the distance assigned to the surface's point lying at the grip axis for scanning position $n$ is given by:

$$
M(n)=\frac{\sum_{n=1}^{n} \operatorname{Measure}\left(i d \_s\right) \times D c\left(i d \_s\right)}{\sum_{u=1}^{4} D c\left(i d \_s\right)} .
$$

It is worth to note that the Measure values are validated at the SP module of level 3 according to the range of values physically possible. A set of tests is applied to each measure to determine if it corresponds to a point inside or outside the object and to testify if the received value is consistent with the physical set-up. Sensors which measures are not validated do not enter into the average calculation.

Also it should be remarked that each Dc value is in effect a product of 3 partial degrees of confidence

$$
D c\left(i d \_s\right)=D c \_1\left(i d \_s\right) \times D c \_2\left(i d \_s\right) \times D c \_3\left(i d \_s\right)
$$

which heuristically are defined to tackle different aspects of the problems posed by using multiple sonars to measure distance.

$D c_{-} \_$, degree of confidence 1, expresses the alignment of the sensor values to predictable values given by a leastsquares linear predictor/estimator of the surface's inclination.

$D c \_2$, degree of confidence 2, expresses the agreement of each sensor measure with the measures of the other 3 . The underlying assumption is that one is dealing with surfaces of smooth and small curvature.

Finally $D c_{-} 3$ was specifically set-up to account for the detection of the part's edges. Its calculation uses a sigmoid function to approximate the probability distribution that the grip is pointing effectively to the part.

When compared with the previous results obtained for a flat object surface of $20 \times 20 \mathrm{~cm}$, the performance of this process is much better, as Fig. 9 shows. The flat surface was positioned with 20 degrees relatively to the scanning plan. The shape of the object surface and its description were well retrieved.

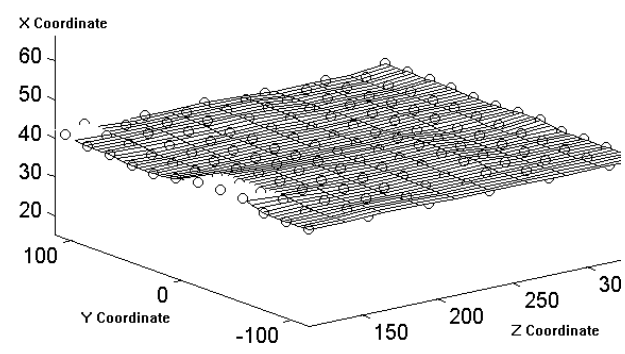

Fig. 9 - Experimental results for the map of a flat rectangular part with averaged measures of the four sonars

\section{CONCLUSIONS AND FUTURE WORK}


The RCS model provides a general conceptual framework for the system's design and software development. It is flexible, powerful and easily integrates diverse notions and work made by different people. It accommodates well the heterogeneous hardware devices envisaged for sensing the geometry of the part to be painted. It showed easy to change the generated behaviours and the interconnection of modules. The use of multiresolution gives a natural dimension for stratifying functionally the system in levels while the partition of each level in the modules depicted follows feedback/feedforward principles for generating system's activity. In our opinion it represents a step towards $\mathrm{M}$. Minsky [12] contention that future intelligent systems will be designed and developed in a component assembly fashion.

Besides the generation of painting trajectories, there is a lot of future work to be done in this project, including improvements to the results already obtained. Current work is in progress for having the grip axis normal to the estimated surface curvature. Also the adaptation of the depth measuring grid resolution to the surface curvature is being worked out.

To minimise the movement duration of the manipulator, direct control of the joints is being considered, eventually with non-linear techniques. In what respects the geometry's acquisition time, high level techniques as clustering shapes and the correspondent class creation, as well as learning on geometry is being envisaged. All these moves find a natural framing in the reference model adopted.

\section{REFERENCES}

[1] J.P. Vanbremeersch, J. Chandler and A. C. Ehresmann, "Are interactions between different timescales a characteristic of complexity?", Proceedings of the E-C-H-O Symposium, Université de Picardie Jules Verne, Amiens (France), August, 1996, pp. 2123.

[2] J.S. Albus, "Outline for a Theory of Intelligence". IEEE Transactions on Systems, Man and Cybernetics, no. 3, May/June 1991,p. 21.

[3] J.S. Albus, "RCS: A Reference Model Architecture for Intelligent Control," IEEE Special Issue of Computer entitled Computer Architectures for Intelligent Machines, May 1992.

[4] J.S. Albus and A.M. Meystel, "A Reference Model Architecture for Design and Implementation of Intelligent Control in Large and Complex Systems". International Journal of Intelligent Control and Systems, vol. 1, no. 1, March 1996, p. 15-30.

[5] N. Otsu, "A threshold selection method from grey level histogram". IEEE Transactions on Pattern Analysis and Machine Intelligence, vol. SMC-8, 1978.

[6] R. Lima and A. Campilho, "Thresholding selection methods: a comparative study". Proceedings of the RECPAD'94 - The $6^{\text {th }}$ Annual Conference of the
Portuguese Association for Pattern Recognition, 1994.

[7] R. Gonzalez and R. Woods, Digital Image Processing. Addison Wesley, 1992.

[8] G. Blais and M.D. Levine, "Registering Multiview Range Data to Create 3D Computer Objects". IEEE Transactions on Pattern Analysis and Machine Intelligence, 17 (8) p.820-824.

[9] Foley and Hughes, 3D Computer Graphics \& Principle, Addison Wesley, 1992.

[10] A. Watt, 3D Computer Graphics, Addison Wesley, 1993.

[11] E. Piat and D. Meizel, " Range Data Fusion for occupancy grid building," in Proceedings of the $4^{\text {th }}$ International Symposium on Intelligent Robotic Systems '96, pp. 353-360, Lisbon 1996.

[12] M. Minsky, "Logical vs. Analogical or Symbolic vs. Connectionist or Neat vs. Scruffy". Artificial Intelligence at MIT.. Expanding Frontiers, Patrick H. Winston (Ed.), no. 1, MIT Press, 1990. 